\title{
Supersymmetric Extension of the Snyder Algebra
}

\author{
L. GoubA* and A. Stern ** \\ *The Abdus Salam International Centre for Theoretical Physics(ICTP) \\ Strada Costiera 11, 34014, Trieste, Italy. Email: lgouba@ictp.it \\ ** Dept. of Physics and Astronomy, Univ. of Alabama, \\ Tuscaloosa, Al 35487, U.S.A. Email: astern@bama.ua.edu
}

\begin{abstract}
We obtain a minimal supersymmetric extension of the Snyder algebra and study its representations. The construction differs from the general approach given in Hatsuda and Siegel (hep-th/0311002) and does not utilize super-de Sitter groups. The spectra of the position operators are discrete, implying a lattice description of space, and the lattice is compatible with supersymmetry transformations.
\end{abstract}




\section{Introduction}

Snyder showed long ago that the continuous symmetries of space-time can be made consistent with a lattice through the construction of a covariant noncommutative algebra.[1] The algebra that Snyder proposed is a nontrivial unification of space-time with the Poincaré algebra. The lattice is a result of the discrete representations of Snyder's algebra. It is not a classical lattice because only one spatial coordinate can be determined in a measurement. Here we generalize the Snyder algebra to get a nontrivial unification of space-time with the super-Poincaré algebra. Our supersymmetric extension is minimal and also has discrete representations corresponding to a spatial lattice. The lattice is compatible with supersymmetry transformations, and the lattice spacing is half that of the bosonic case. The supersymmetry generators act in a nonstandard way on the space-time. As discussed in [2], the translation group generated by the momenta is not associated with discrete translations on the spatial lattice. The system presented here might be useful in formulating an alternative discretization of supersymmetric field theories, where supersymmetry transformations are consistently implemented on the lattice.[3] Supersymmetry has been shown useful in improving renormalizability properties of noncommutative field theories, [4] and this may turn out to be the case for the Snyder model as well. We shall not examine field theory here, but note that the lattice appears to be the appropriate setting for studying field theory in this case.

Supersymmetric extensions of the Snyder algebra in arbitrary space-time dimensions were previously constructed by Hatsuda and Siegel.[5] Their general approach is based on supersymmetric de Sitter algebras. [6] We shall give an alternative construction which attaches a pair of Grassmann odd spinors to de Sitter space, but does not require the spinors to generate a supersymmetric de Sitter algebra. Nevertheless, the super-Poincaré algebra is recovered upon projecting to Minkowski space-time. The algebra combines $N=1$ supersymmetry with the Snyder algebra, and in contrast to [5], the fermionic coordinates are all anticommuting.

The Snyder algebra and its supersymmetric extensions are characterized by a deformation parameter $\Lambda$, which is proportional to one over the lattice spacing. One can define the action of the supersymmetry generators on superspace in the $\Lambda \rightarrow \infty$ limit. Superspace cannot be defined for finite $\Lambda$ (except in one-dimension), since the operators associated with the spacetime coordinates are not simultaneously diagonalizable. On the other hand, the momentum operators commute, and so we can define a 'super-momentum space' for finite $\Lambda$. We shall introduce fields on this space and write down differential representations for the supersymmetry generators.

Discrete representations of the Snyder algebra were examined in [2], where it was argued that there are two distinct Hilbert spaces. (This is a result of the projection from de Sitter space.) The two are distinguished by $S U(2)$ quantum numbers, which are integer in one case and half-integer in the other. In our supersymmetric extension of the model we can construct fermionic raising and lowering operators that combine the integer and half-integer states in 
a single graded space. The raising and lowering operators simultaneously change the spin by $\pm 1 / 2$, and the location on the lattice to the nearest neighbor. Care must be taken in defining an involution for the fermionic operators. Negative norm states result if complex conjugation connects Lorentz spinors in the standard way. On the other hand, negative norm states are absent upon adopting an alternative involution which maps one de Sitter spinor to the other.

The outline of this article is as follows: In section 2 we review the derivation of the Snyder algebra starting from de Sitter space. De Sitter spinors are introduced in section 3. Their projection to Minkowski space yields the minimal supersymmetric Snyder algebra. Discrete representations are examined in section 4 and an involution is introduced in section 5 which eliminates negative norm states. A differential representation for the supersymmetry generators on super-momentum space is given in section 6. Concluding remarks are made in section 7 , including the construction of the extended supersymmetric Snyder algebra.

\section{Snyder algebra}

The original derivation of the Snyder algebra starts with a de Sitter manifold. Say that the latter is coordinatized by $P_{M}, \quad M=0,1,2,3,4$, which are all commuting and subject to the constraint

$$
P_{M} P_{N} \eta_{\mathrm{dS}}^{M N}=1
$$

where $\eta_{\mathrm{dS}}$ is the de Sitter metric $\eta_{\mathrm{dS}}=\operatorname{diag}(-1,1,1,1,1)$. We take $P_{M}$ to be dimensionless. Denote by $\ell^{M N}=-\ell^{N M}$ the generators of the de Sitter group with commutation relations

$$
\left[\ell^{M N}, \ell^{R S}\right]=i\left(\eta_{\mathrm{dS}}^{R M} \ell^{N S}-\eta_{\mathrm{dS}}^{R N} \ell^{M S}+\eta_{\mathrm{dS}}^{S M} \ell^{R N}-\eta_{\mathrm{dS}}^{S N} \ell^{R M}\right)
$$

Assuming $P_{M}$ transforms as a five-vector with respect to the de Sitter group, one has

$$
\left[\ell^{M N}, P^{R}\right]=i\left(\eta_{\mathrm{dS}}^{R M} P^{N}-\eta_{\mathrm{dS}}^{R N} P^{M}\right)
$$

The projection of this system to four dimensions was obtained by defining the four-momentum vector $p^{m}, m=0,1,2,3$, according to

$$
p^{m}=\Lambda \frac{P^{m}}{P^{4}},
$$

and identifying $\ell^{4 m}$ with the space-time four vector $x^{m}$, up to the dimensionful parameter $\Lambda$. For this Snyder could consistently set

$$
\ell^{m n}=x^{m} p^{n}-x^{n} p^{m} \quad \ell^{4 m}=\Lambda x^{m}
$$

and then derive the algebra for the two Lorentz vectors from (2.2) and (2.3),

$$
\left[x^{m}, x^{n}\right]=\frac{i}{\Lambda^{2}} \ell^{m n} \quad\left[x^{m}, p^{n}\right]=i\left(\eta^{m n}+\frac{p^{m} p^{n}}{\Lambda^{2}}\right) \quad\left[p^{m}, p^{n}\right]=0,
$$


where $\eta=\operatorname{diag}(-1,1,1,1)$ is the Minkowski metric. $\Lambda$ is a deformation parameter, and the Heisenberg algebra is recovered in the limit $\Lambda \rightarrow \infty$. From (2.1) and (2.4) one gets a mass upper bound, $-p^{m} p_{m} \leq \Lambda^{2}$. The spatial coordinates $x_{i}$, along with the orbital angular momentum $\ell^{i j}, i, j, \ldots=1,2,3$, generate the $S O(4)$ subgroup of the de Sitter group. The discrete spectra of the position operators follows from the discrete representations of $S O(4)$.

\section{Minimal supersymmetric extension}

To get the supersymmetric version of the algebra we attach two spinor degrees of freedom to de Sitter space. Define $\Theta_{\mathrm{A}}$ and $\bar{\Theta}_{\mathrm{A}}, \mathrm{A}=1,2,3,4$, to be two conjugate four-dimensional spinors which commute with $P^{M}$ and $\ell^{M N}$, and satisfy the anti-commutation relations

$$
\left\{\Theta_{\mathrm{A}}, \bar{\Theta}_{\mathrm{B}}\right\}=-i \delta_{\mathrm{AB}} \quad\left\{\Theta_{\mathrm{A}}, \Theta_{\mathrm{B}}\right\}=\left\{\bar{\Theta}_{\mathrm{A}}, \bar{\Theta}_{\mathrm{B}}\right\}=0
$$

Assume they give a spin contribution to the generators of the de Sitter group. Denote by $j^{M N}=-j^{N M}$ the sum of the orbital and spin contributions,

$$
j^{M N}=\ell^{M N}-\bar{\Theta} \Sigma^{M N} \Theta,
$$

where $\Sigma^{M N}=-\Sigma^{N M}$ define the four-by-four spinor representation of the algebra

$$
\left[\Sigma^{M N}, \Sigma^{R S}\right]=\eta_{\mathrm{dS}}^{R M} \Sigma^{N S}-\eta_{\mathrm{dS}}^{R N} \Sigma^{M S}+\eta_{\mathrm{dS}}^{S M} \Sigma^{R N}-\eta_{\mathrm{dS}}^{S N} \Sigma^{R M}
$$

Then $\Theta$ and $\bar{\Theta}$ transform as de Sitter spinors, while $P^{M}$ remains a five-vector under de Sitter transformations, now generated by $j^{M N}$,

$$
\begin{aligned}
{\left[j^{M N}, \bar{\Theta}\right] } & =i \bar{\Theta} \Sigma^{M N} \\
{\left[j^{M N}, \Theta\right] } & =-i \Sigma^{M N} \Theta \\
{\left[j^{M N}, P^{R}\right] } & =i\left(\eta_{\mathrm{dS}}^{R M} P^{N}-\eta_{\mathrm{dS}}^{R N} P^{M}\right) \\
{\left[j^{M N}, j^{R S}\right] } & =i\left(\eta_{\mathrm{dS}}^{R M} j^{N S}-\eta_{\mathrm{dS}}^{R N} j^{M S}+\eta_{\mathrm{dS}}^{S M} j^{R N}-\eta_{\mathrm{dS}}^{S N} j^{R M}\right)
\end{aligned}
$$

To project to four space-time dimensions we again assume (2.4) and (2.5), leading to the Snyder algebra (2.6) for the bosonic operators $x^{m}$ and $p^{m}$. For the spinor representations, we choose

$$
\Sigma^{m n}=\left(\begin{array}{cc}
\sigma^{m n} & \\
& \bar{\sigma}^{m n}
\end{array}\right) \quad \Sigma^{4 m}=\frac{1}{2}\left(\begin{array}{cc}
\sigma^{m} \\
\bar{\sigma}^{m}
\end{array}\right),
$$

where we follow conventions in Wess and Bagger:[7] $\sigma^{0}=\bar{\sigma}^{0}=-1_{2 \times 2}$ and $\bar{\sigma}^{\mathrm{i}}=-\sigma^{\mathrm{i}}$, where $\sigma^{\mathrm{i}}, \mathbf{i}=1,2,3$, are the three Pauli matrices. The $2 \times 2$ Lorentz matrices $\sigma^{m n}$ and $\bar{\sigma}^{m n}$ are defined by

$$
\sigma^{m n}=\frac{1}{4}\left(\sigma^{m} \bar{\sigma}^{n}-\sigma^{n} \bar{\sigma}^{m}\right) \quad \bar{\sigma}^{m n}=\frac{1}{4}\left(\bar{\sigma}^{m} \sigma^{n}-\bar{\sigma}^{n} \sigma^{m}\right)
$$


They satisfy the identities

$$
\begin{aligned}
2 \sigma^{m n} \sigma^{s} & =\eta^{m s} \sigma^{n}-\eta^{n s} \sigma^{m}+i \epsilon^{m n s r} \sigma_{r} \\
2 \sigma^{s} \bar{\sigma}^{m n} & =-\eta^{m s} \sigma^{n}+\eta^{n s} \sigma^{m}+i \epsilon^{m n s r} \sigma_{r} \\
2 \bar{\sigma}^{m n} \bar{\sigma}^{s} & =\eta^{m s} \bar{\sigma}^{n}-\eta^{n s} \bar{\sigma}^{m}-i \epsilon^{m n s r} \bar{\sigma}_{r} \\
2 \bar{\sigma}^{s} \sigma^{m n} & =-\eta^{m s} \bar{\sigma}^{n}+\eta^{n s} \bar{\sigma}^{m}-i \epsilon^{m n s r} \bar{\sigma}_{r} \\
{\left[\sigma^{m n}, \sigma^{r s}\right] } & =\eta^{r m} \sigma^{n s}-\eta^{r n} \sigma^{m s}+\eta^{s m} \sigma^{r n}-\eta^{s n} \sigma^{r m} \\
{\left[\bar{\sigma}^{m n}, \bar{\sigma}^{r s}\right] } & =\eta^{r m} \bar{\sigma}^{n s}-\eta^{r n} \bar{\sigma}^{m s}+\eta^{s m} \bar{\sigma}^{r n}-\eta^{s n} \bar{\sigma}^{n m}
\end{aligned}
$$

Next we express the two four-dimensional spinors $\Theta$ and $\bar{\Theta}$ in terms of four two-dimensional Lorentz spinors $Q_{\alpha}, \bar{Q}_{\dot{\alpha}}, \theta^{\alpha}$ and $\bar{\theta}^{\dot{\alpha}}, \alpha, \dot{\alpha}=1,2$. We write $\bar{\Theta}$ as a row matrix and $\Theta$ as a column matrix according to

$$
\bar{\Theta}=\frac{1}{\sqrt{\Lambda}}\left(\begin{array}{lll}
\Lambda \theta & \bar{Q}+i \theta \sigma^{n} p_{n}
\end{array}\right) \quad \Theta=\frac{1}{\sqrt{\Lambda}}\left(\begin{array}{c}
-Q+i p_{n} \sigma^{n} \bar{\theta} \\
\Lambda \bar{\theta}
\end{array}\right)
$$

$\Lambda$ being the same dimensionful parameter appearing in (2.4). Since $\Theta$ and $\bar{\Theta}$ commute with $p^{m}$, the four two-dimensional spinors must also commute with $p^{m}$. From the anti-commutation relations (3.1) for $\Theta$ and $\bar{\Theta}$, it follows:

$i)$ that $\theta^{\alpha}$ and $\bar{\theta}^{\dot{\alpha}}$ are all anti-commuting Grassmann coordinates* , i.e.,

$$
\left\{\theta^{\alpha}, \theta^{\beta}\right\}=\left\{\bar{\theta}^{\dot{\alpha}}, \bar{\theta}^{\dot{\beta}}\right\}=\left\{\theta^{\alpha}, \bar{\theta}^{\dot{\beta}}\right\}=0,
$$

ii) that $Q_{\alpha}$ and $\bar{Q}_{\dot{\alpha}}$ are canonically conjugate to $\theta^{\alpha}$ and $\bar{\theta}^{\dot{\alpha}}$, respectively,

$$
\left\{Q_{\alpha}, \theta^{\beta}\right\}=i \delta_{\alpha}^{\beta} \quad\left\{\bar{Q}_{\dot{\alpha}}, \bar{\theta}^{\dot{\beta}}\right\}=-i \delta_{\dot{\alpha}}^{\dot{\beta}} \quad\left\{Q_{\alpha}, \bar{\theta}^{\dot{\beta}}\right\}=\left\{\bar{Q}_{\dot{\alpha}}, \theta^{\beta}\right\}=0,
$$

and $i i i)$ that $Q_{\alpha}$ and $\bar{Q}_{\dot{\alpha}}$ are super-translation generators,

$$
\left\{Q_{\alpha}, \bar{Q}_{\dot{\alpha}}\right\}=2 \sigma_{\alpha \dot{\alpha}}{ }^{m} p_{m} \quad\left\{Q_{\alpha}, Q_{\beta}\right\}=\left\{\bar{Q}_{\dot{\alpha}}, \bar{Q}_{\dot{\beta}}\right\}=0
$$

Finally, since $\Theta$ and $\bar{\Theta}$ commute with coordinates $x^{m}$, it follows:

$i v)$ that $\theta$ and $\bar{\theta}$ also commute with $x^{m}$, while

$$
\left[Q_{\alpha}, x^{m}\right]=\left(\sigma^{m}+\frac{\sigma^{n} p_{n} p^{m}}{\Lambda^{2}}\right)_{\alpha \dot{\alpha}} \bar{\theta}^{\dot{\alpha}} \quad\left[\bar{Q}_{\dot{\alpha}}, x^{m}\right]=-\theta^{\alpha}\left(\sigma^{m}+\frac{\sigma^{n} p_{n} p^{m}}{\Lambda^{2}}\right)_{\alpha \dot{\alpha}}
$$

${ }^{*}$ This is in contrast to [5], where the anticommutators of fermionic coordinates are in general a linear combination of space-time coordinates, Lorentz generators and additional $S O(N)$ generators. 
$Q_{\alpha}, \bar{Q}_{\dot{\alpha}}$ and $p^{m}$ generate the $N=1$ super-translation group. Upon including the Lorentz generators $j^{m n}$, which using (3.8) can be expressed as

$$
j^{m n}=x^{m} p^{n}-x^{n} p^{m}+\theta \sigma^{m n} Q-\bar{Q} \bar{\sigma}^{m n} \bar{\theta}-\epsilon^{m n r s}\left(\theta \sigma_{r} \bar{\theta}\right) p_{s},
$$

we get the super-Poincaré group. From the commutation relations with $j^{m n}$,

$$
\begin{aligned}
{\left[Q_{\alpha}, j^{m n}\right]=i\left(\sigma^{m n} Q\right)_{\alpha} } & {\left[\bar{Q}_{\dot{\alpha}}, j^{m n}\right]=-i\left(\bar{Q} \bar{\sigma}^{m n}\right)_{\dot{\alpha}} } \\
{\left[\theta^{\alpha}, j^{m n}\right]=-i\left(\theta \sigma^{m n}\right)^{\alpha} } & {\left[\bar{\theta}^{\dot{\alpha}}, j^{m n}\right]=i\left(\bar{\sigma}^{m n} \bar{\theta}\right)^{\dot{\alpha}} } \\
{\left[x^{r}, j^{m n}\right]=i\left(x^{m} \eta^{r n}-x^{n} \eta^{r m}\right) } & {\left[p^{r}, j^{m n}\right]=i\left(p^{m} \eta^{r n}-p^{n} \eta^{r m}\right), }
\end{aligned}
$$

it follows that $Q_{\alpha}, \bar{Q}_{\dot{\alpha}}, \theta^{\alpha}$ and $\bar{\theta}^{\dot{\alpha}}$ transform as Lorentz spinors, while $x^{m}$ and $p^{m}$ as Lorentz vectors.

Eqs. (3.9)-(3.12), along with (2.6), defines a minimal supersymmetric extension of the Snyder algebra. It is expressed above in terms of $\theta^{\alpha}, \bar{\theta}^{\dot{\alpha}}$ and $x^{m}$, in addition to the $N=1$ super-translation generators $Q_{\alpha}, \bar{Q}_{\dot{\alpha}}$ and $p^{m}$. Alternatively, instead of $x^{m}$, we can define the following space-time coordinates in the supersymmetric theory:

$$
\begin{aligned}
X^{m}=\frac{1}{\Lambda} j^{4 m}=x^{m}+ & \frac{1}{2 \Lambda^{2}}\left(\bar{Q} \bar{\sigma}^{m} Q+i \theta \sigma^{n} p_{n} \bar{\sigma}^{m} Q-i \bar{Q} \bar{\sigma}^{m} \sigma^{n} p_{n} \bar{\theta}\right) \\
& -\frac{1}{\Lambda^{2}} p^{m} \theta \sigma^{n} p_{n} \bar{\theta}-\frac{1}{2}\left(1-\frac{p^{n} p_{n}}{\Lambda^{2}}\right) \theta \sigma^{m} \bar{\theta}
\end{aligned}
$$

It forms a closed algebra with the Poincaré generators. In contrast to (2.6), one gets

$$
\left[X^{m}, X^{n}\right]=\frac{i}{\Lambda^{2}} j^{m n} \quad\left[X^{m}, p^{n}\right]=i\left(\eta^{m n}+\frac{p^{m} p^{n}}{\Lambda^{2}}\right) \quad\left[p^{m}, p^{n}\right]=0,
$$

where $j^{m n}$ are the Lorentz generators (3.13). The latter can be re-expressed in terms of $X^{n}$ using (3.15). $X^{n}$ 's commutators with the spinors are given by

$$
\begin{aligned}
{\left[X^{m}, \theta\right] } & =\frac{i}{2 \Lambda^{2}}\left(\bar{Q}+i \theta \sigma^{n} p_{n}\right) \bar{\sigma}^{m} \\
{\left[X^{m}, \bar{\theta}\right] } & =\frac{i}{2 \Lambda^{2}} \bar{\sigma}^{m}\left(Q-i p_{n} \sigma^{n} \bar{\theta}\right) \\
{\left[X^{m}, \bar{Q}+i \theta \sigma^{n} p_{n}\right] } & =\frac{i}{2} \theta \sigma^{m} \\
{\left[X^{m}, Q-i \sigma^{n} p_{n} \bar{\theta}\right] } & =\frac{i}{2} \sigma^{m} \bar{\theta}
\end{aligned}
$$

Eqs. (3.16) and (3.17), along with (3.9)-(3.11), give an alternative definition of the supersymmetric Snyder algebra. Due to the nonstandard commutation relations of $Q_{\alpha}, \bar{Q}_{\dot{\alpha}}$ and $p_{m}$ with the coordinate operators $X^{m}$ (or $x^{m}$ ), the super-translation group acts in a nonstandard fashion on space-time. 


\section{Discrete representations}

We regard $X_{i}, i=1,2,3$, as the position operators for the supersymmetric Snyder algebra. They are the spatial components of $X^{n}$. From (3.16), $X_{i}$, along with $j^{i j}$, generate another $S O(4)$ group. It follows that $X_{i}$, like $x_{i}$, have discrete spectra. Here we can define

$$
A_{\mathrm{i}}=\frac{1}{2}\left(J_{\mathrm{i}}+\Lambda X_{\mathrm{i}}\right) \quad B_{\mathrm{i}}=\frac{1}{2}\left(J_{\mathrm{i}}-\Lambda X_{\mathrm{i}}\right),
$$

where $J_{i}=\frac{1}{2} \epsilon_{\mathrm{ijk}} j^{\mathrm{jk}}$ is the rotation generator. They satisfy two $s u(2)$ algebras

$$
\begin{aligned}
& {\left[A_{\mathrm{i}}, A_{\mathrm{j}}\right]=i \epsilon_{\mathrm{ijk}} A_{\mathrm{k}}} \\
& {\left[B_{\mathrm{i}}, B_{\mathrm{j}}\right]=i \epsilon_{\mathrm{ijk}} B_{\mathrm{k}}} \\
& {\left[A_{\mathrm{i}}, B_{\mathrm{j}}\right]=0}
\end{aligned}
$$

$A_{\mathrm{i}} A_{\mathrm{i}}, B_{\mathrm{i}} B_{\mathrm{i}}, A_{3}$ and $B_{3}$ form a complete set of commuting operators. ${ }^{\dagger}$ We denote their eigenvectors by $\mid j_{A}, j_{B}, m_{A}, m_{B}>$,

$$
\begin{aligned}
A_{\mathrm{i}} A_{\mathrm{i}} \mid j_{A}, j_{B}, m_{A}, m_{B}> & =j_{A}\left(j_{A}+1\right) \mid j_{A}, j_{B}, m_{A}, m_{B}> \\
B_{\mathrm{i}} B_{\mathrm{i}} \mid j_{A}, j_{B}, m_{A}, m_{B}> & =j_{B}\left(j_{B}+1\right) \mid j_{A}, j_{B}, m_{A}, m_{B}> \\
A_{3} \mid j_{A}, j_{B}, m_{A}, m_{B}> & =m_{A} \mid j_{A}, j_{B}, m_{A}, m_{B}> \\
B_{3} \mid j_{A}, j_{B}, m_{A}, m_{B}> & =m_{B} \mid j_{A}, j_{B}, m_{A}, m_{B}>,
\end{aligned}
$$

where $m_{A}=-j_{A}, 1-j_{A}, \ldots, j_{A}$, and $m_{B}=-j_{B}, 1-j_{B}, \ldots, j_{B} . j_{A}$ and $j_{B}$ take values $0, \frac{1}{2}, 1, \frac{3}{2}, \ldots$, and label the $S O(4)$ representations. $\mid j_{A}, j_{B}, m_{A}, m_{B}>$ is also an eigenvector of $J_{3}$ and $X_{3}$, whose corresponding eigenvalues are $m_{A}+m_{B}$ and $\left(m_{A}-m_{B}\right) / \Lambda$, respectively. Applying $A_{ \pm}$or $B_{ \pm}$changes $\left|J_{3}\right|$ by 1 and $\left|X_{3}\right|$ by $1 / \Lambda$.

$S O(4)$ group representations are also present in the non-supersymmetric version of the theory, and there $m_{A}$ and $m_{B}$ are either both integer or both half-integer.[2] It followed that the associated (orbital) angular momentum operators had integer eigenvalues, and also that the eigenvalues of the position operators $x_{i}$ were evenly spaced at intervals of $\Lambda^{-1}$. We show below that for the supersymmetric Snyder algebra, eigenvalues of the position operators $X_{i}$ are evenly spaced at intervals of $\frac{1}{2} \Lambda^{-1}$, and that both integer and half-integer values of $m_{A}$ and $m_{B}$ occur in the representation of the graded algebra. This means that both integer and half-integer values for $j_{A}$ and $j_{B}$ occur in a representation of the supersymmetry algebra.

From (3.14) and (3.17) one can construct various linear combinations of the spinors which act as raising and lowering operators with respect to the eigenvalues of the operators $X_{\mathrm{i}}$ and

\footnotetext{
${ }^{\dagger}$ The Casimir operators $A_{\mathrm{i}} A_{\mathrm{i}}$ and $B_{\mathrm{i}} B_{\mathrm{i}}$ are independent, unlike their counterparts in the non supersymmetric theory. [2]
} 
$J_{\dot{i}}$, or equivalently, $A_{\dot{i}}$ and $B_{\dot{i}}$. For the case of $\mathrm{i}=3$, we can define the raising and lowering operators $a_{ \pm}^{\mathrm{A}}, \mathrm{A}=1,2,3,4$, according to

$$
\begin{aligned}
& \sqrt{2} a_{ \pm}^{1}=\bar{\Theta}_{1} \mp i \bar{\Theta}_{3}=\sqrt{\Lambda} \theta^{1} \mp \frac{i}{\sqrt{\Lambda}}\left(\bar{Q}+i \theta \sigma^{n} p_{n}\right)_{1} \\
& \sqrt{2} a_{ \pm}^{2}=\bar{\Theta}_{2} \pm i \bar{\Theta}_{4}=\sqrt{\Lambda} \theta^{2} \pm \frac{i}{\sqrt{\Lambda}}\left(\bar{Q}+i \theta \sigma^{n} p_{n}\right)_{2} \\
& \sqrt{2} a_{ \pm}^{3}=\Theta_{3} \pm i \Theta_{1}=\sqrt{\Lambda} \bar{\theta}^{1} \mp \frac{i}{\sqrt{\Lambda}}\left(Q-i \sigma^{n} p_{n} \bar{\theta}\right)_{1} \\
& \sqrt{2} a_{ \pm}^{4}=\Theta_{4} \mp i \Theta_{2}=\sqrt{\Lambda} \bar{\theta}^{2} \pm \frac{i}{\sqrt{\Lambda}}\left(Q-i \sigma^{n} p_{n} \bar{\theta}\right)_{2}
\end{aligned}
$$

Their nonvanishing anticommutators are

$$
\left\{a_{\mp}^{1}, a_{ \pm}^{3}\right\}=\left\{a_{ \pm}^{2}, a_{\mp}^{4}\right\}= \pm 1
$$

From (3.14) and (3.17) one has

$$
\begin{gathered}
{\left[X_{3}, a_{ \pm}^{\mathrm{A}}\right]= \pm \frac{1}{2 \Lambda} a_{ \pm}^{\mathrm{A}}} \\
{\left[J_{3}, a_{ \pm}^{1,4}\right]=\frac{1}{2} a_{ \pm}^{1,4} \quad\left[J_{3}, a_{ \pm}^{2,3}\right]=-\frac{1}{2} a_{ \pm}^{2,3}}
\end{gathered}
$$

Say that $\mid m_{A}, m_{B}>$ is an eigenvector of $X_{3}$ and $J_{3}$ with eigenvalue is $\left(m_{A}-m_{B}\right) / \Lambda$ and $m_{A}+m_{B}$, respectively, where for convenience we ignore the dependence on the indices $j_{A}$ and $j_{B}$. Then $a_{ \pm}^{\mathrm{A}} \mid m_{A}, m_{B}>$ are also eigenvectors of $X_{3}$ and $J_{3}$. From (4.6), the $X_{3}$ eigenvalue of $a_{ \pm}^{\mathrm{A}} \mid m_{A}, m_{B}>$ is $\left(m_{A}-m_{B} \pm \frac{1}{2}\right) / \Lambda$. From (4.7), the $J_{3}$ eigenvalue of $a_{ \pm}^{1,4} \mid m_{A}, m_{B}>$ is $m_{A}+m_{B}+\frac{1}{2}$, while the $J_{3}$ eigenvalue of $a_{ \pm}^{2,3} \mid m_{A}, m_{B}>$ is $m_{A}+m_{B}-\frac{1}{2}$. So application of $a_{ \pm}^{\text {A }}$ simultaneously changes $\left|J_{3}\right|$ by $\frac{1}{2}$ and $\left|X_{3}\right|$ by $\frac{1}{2 \Lambda}$. Up to degenerate states,

$$
\begin{aligned}
a_{+}^{1,4} \mid m_{A}, m_{B}> & \sim\left|m_{A}+\frac{1}{2}, m_{B}\right\rangle \\
a_{-}^{1,4} \mid m_{A}, m_{B}> & \sim\left|m_{A}, m_{B}+\frac{1}{2}\right\rangle \\
a_{+}^{2,3} \mid m_{A}, m_{B}> & \sim\left|m_{A}, m_{B}-\frac{1}{2}\right\rangle \\
a_{-}^{2,3} \mid m_{A}, m_{B}> & \sim\left|m_{A}-\frac{1}{2}, m_{B}\right\rangle
\end{aligned}
$$

It follows that, unlike what happens in the non-supersymmetric theory, both integer and halfinteger values of $m_{A}$ and $m_{B}$ occur in the representations of the supersymmetric algebra. Consequently, both integer and half-integer values of $j_{A}$ and $j_{B}$ occur in the representations. In the non-supersymmetric theory, the eigenvalues of the position operator are regularly spaced at intervals of $\Lambda^{-1}$, whereas here they are spaced at intervals of $\frac{1}{2} \Lambda^{-1}$. $a_{ \pm}^{\mathrm{A}}$ were defined to raise and lower eigenvalues associated with the 3 -direction. Raising and lowering operators can also be constructed for the 1 - and 2-directions, and they yield the same spectra for the position and angular momentum operators. 


\section{Involutions}

There are two approaches to introducing an involution, or complex conjugation, of the algebra. The first which we discuss below connects Lorentz spinor $\theta$ with $\bar{\theta}$ and $Q$ with $\bar{Q}$ in the usual way. It leads to negative norm states. No negative norm states result from an alternative involution. The latter maps one de Sitter spinor to the other.

Complex conjugation (which we denote by $*$ ) in four space-time dimensions standardly relates spinor representations to conjugate representations according to

$$
\left(\theta^{\alpha}\right)^{*}=\bar{\theta}^{\dot{\alpha}} \quad\left(Q_{\alpha}\right)^{*}=\bar{Q}_{\dot{\alpha}}
$$

Its action on the de Sitter spinors $\Theta$ and $\bar{\Theta}$ is then

$$
\left(\bar{\Theta}^{*}\right)=\left(\begin{array}{rr} 
& 11 \\
-1 l &
\end{array}\right) \Theta \quad\left(\Theta^{*}\right)=\bar{\Theta}\left(\begin{array}{rr}
11 \\
-11 &
\end{array}\right)
$$

It is then easy to show that

$$
\left\{\Theta_{\mathrm{A}}^{*}, \bar{\Theta}_{\mathrm{B}}^{*}\right\}=i \delta_{\mathrm{AB}} \quad\left\{\Theta_{\mathrm{A}}^{*}, \Theta_{\mathrm{B}}^{*}\right\}=\left\{\bar{\Theta}_{\mathrm{A}}^{*}, \bar{\Theta}_{\mathrm{B}}^{*}\right\}=0
$$

This means that complex is consistent with the supersymmetric Snyder algebra generated by $x^{m}, p_{m}, \theta^{\alpha}, \bar{\theta}^{\dot{\alpha}}, Q_{\alpha}$ and $\bar{Q}_{\dot{\alpha}}$, since all anticommutators between Lorentz spinors followed from (3.1). We assume that $p_{m}$ and $x^{m}$ are real. It follows that all of the de Sitter group generators $X^{m}$ and $j^{m n}$ are also real.

For the raising and lowering operators we get

$$
\left(a_{ \pm}^{1}\right)^{*}=a_{\mp}^{3} \quad\left(a_{ \pm}^{2}\right)^{*}=a_{\mp}^{4}
$$

From the anticommutation relations (4.5), the norm-squared of eigenvectors of $A_{3}$ and $B_{3}$ are related by

$$
\begin{aligned}
& \left|a_{+}^{1}\right| m_{A}, m_{B}>\left.\right|^{2}=-\left|a_{-}^{3}\right| m_{A}, m_{B}>\left.\right|^{2}-|| m_{A}, m_{B}>\left.\right|^{2} \\
& \left|a_{-}^{2}\right| m_{A}, m_{B}>\left.\right|^{2}=-\left|a_{+}^{4}\right| m_{A}, m_{B}>\left.\right|^{2}-|| m_{A}, m_{B}>\left.\right|^{2}
\end{aligned}
$$

implying the existence of negative norm states. Alternatively, one can define

$$
b_{-}^{\mathrm{A}}=\bar{\Theta}_{\mathrm{A}} \quad b_{+}^{\mathrm{A}}=i \Theta_{\mathrm{A}},
$$

which satisfy the usual algebra of fermionic creation and annihilation operators,

$$
\left\{b_{+}^{\mathrm{A}}, b_{-}^{\mathrm{B}}\right\}=\delta^{\mathrm{AB}} \quad\left\{b_{+}^{\mathrm{A}}, b_{+}^{\mathrm{B}}\right\}=\left\{b_{-}^{\mathrm{A}}, b_{-}^{\mathrm{B}}\right\}=0
$$

However from $(5.2), b_{+}^{\mathrm{A}}$ is not the complex conjugate of $b_{-}^{\mathrm{A}}$, again implying the existence of negative norm states, e.g. $\left|b_{-}^{1}\right| m_{A}, m_{B}>\left.\right|^{2}=-\left|b_{+}^{3}\right| m_{A}, m_{B}>\left.\right|^{2}$. We note from (4.8), that while $b_{ \pm}^{\mathrm{A}}$ acting on $\mid m_{A}, m_{B}>$ is an eigenvector of $J_{3}$, it is not an eigenvector of $X_{3}, A_{3}$ or $B_{3}$. 
On the other hand, negative norm states are absent if we replace the $*$ by another involutionoperation, which we denote by $\star$, satisfying $\left(x^{\star}\right)^{\star}=x,(x y)^{\star}=y^{\star} x^{\star}$ and $i^{\star}=-i$. We define it in terms of the de Sitter spinors according to

$$
\bar{\Theta}_{\mathrm{A}}^{\star}=i \Theta_{\mathrm{A}},
$$

in contrast to $(5.2)$. (5.3) is again satisfied (with $\star$ now replacing $*$ ), and so the $\star$ involution is consistent with the supersymmetric Snyder algebra. We assume that $p_{m}$ and $x^{m}$ are real with respect to $\star$. In order that the de Sitter generators $X^{m}$ and $j^{m n}$ are real under the $\star$ involution we need that

$$
\sigma^{m \star}=-\bar{\sigma}^{m} \quad \bar{\sigma}^{m \star}=-\sigma^{m}
$$

The action of the $\star$ involution on Lorentz spinors is more involved than the previous complex conjugation (5.1). Using (3.8) and (5.9), we get

$$
\begin{aligned}
\left(\theta^{\alpha}\right)^{\star} & =-\frac{i}{\Lambda}\left(Q-i \sigma^{n} p_{n} \bar{\theta}\right)_{\alpha} \\
\left(\bar{\theta}^{\dot{\alpha}}\right)^{\star} & =\frac{i}{\Lambda}\left(\bar{Q}+i \theta \sigma^{n} p_{n}\right)_{\dot{\alpha}} \\
Q_{\alpha}^{\star} & =-\frac{1}{\Lambda}\left(\bar{Q} \bar{\sigma}^{n}\right)^{\alpha} p_{n}-i \Lambda \theta^{\alpha}\left(1-\frac{p^{n} p_{n}}{\Lambda^{2}}\right) \\
\bar{Q}_{\dot{\alpha}}^{\star} & =-\frac{1}{\Lambda}\left(\bar{\sigma}^{n} Q\right)^{\dot{\alpha}} p_{n}+i \Lambda \bar{\theta}^{\dot{\alpha}}\left(1-\frac{p^{n} p_{n}}{\Lambda^{2}}\right)
\end{aligned}
$$

From (5.8), the $\star$ involution of the raising and lowering operators is given by

$$
\left(a_{ \pm}^{1}\right)^{\star}=\mp a_{\mp}^{3} \quad\left(a_{ \pm}^{2}\right)^{\star}= \pm a_{\mp}^{4},
$$

in contrast to (5.4), or simply,

$$
\left(b_{ \pm}^{\mathrm{A}}\right)^{\star}=b_{\mp}^{\mathrm{A}}
$$

We can then introduce a set of states $\{\mid c v>\}$, corresponding to Clifford vacuum states , i.e., $b_{-}^{\mathrm{A}} \mid c v>=0$. They should form a representation of the (non-supersymmetric) Snyder algebra, i.e., the algebra generated by the bosonic operators $x_{i}$ and $p_{i}$, since these operators

commute with $b_{ \pm}^{\mathrm{A}}$. Two such infinite-dimensional representations were found in [2]. To the Clifford vacuum we add all states obtained by acting with $b_{+}^{\mathrm{A}}$ to obtain a representation of the supersymmetric theory. There are no negative norm states in this case, because hermitian conjugation is with respect to $\star$, i.e., hermiticity for any vectors $|\psi\rangle$ and $|\phi\rangle$ here means $<\psi\left|\phi>^{\star}=<\phi\right| \psi>$.

\section{Momentum-dependent superfields}

Superspace is standardly coordinatized by space-time coordinates and Grassmann odd variables. This is not possible for finite $\Lambda$, since the space-time coordinates (either $x^{m}$ or $X^{m}$ ) 
do not commute amongst themselves in this case, and moreover, they have discrete spectra. On the other hand, since $\left[p_{m}, p_{n}\right]=0$, we can define a momentum superspace spanned by $p^{m}, \theta^{\alpha}$ and $\bar{\theta}^{\dot{\alpha}}$, and then write down fields on this space. Using $\frac{\partial}{\partial \theta^{\alpha}} \theta^{\beta}=\delta_{\alpha}^{\beta}, \frac{\partial}{\partial \bar{\theta}^{\dot{\alpha}}} \bar{\theta}^{\dot{\beta}}=\delta_{\dot{\alpha}}^{\dot{\beta}}$ and $\frac{\partial}{\partial \theta^{\alpha}} \bar{\theta}^{\dot{\beta}}=\frac{\partial}{\partial \theta^{\dot{\alpha}}} \theta^{\beta}=0$, one can represent the four spinors in (3.8) by

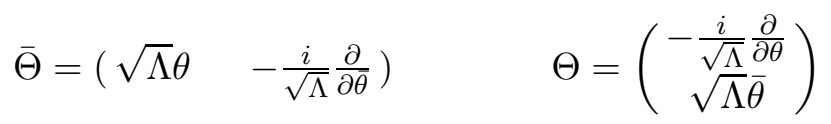

Then $Q_{\alpha}, \bar{Q}_{\dot{\alpha}}, x^{m}$ and $j^{m n}$ are given by differential operators

$$
\begin{aligned}
Q_{\alpha} & =i\left(\frac{\partial}{\partial \theta^{\alpha}}+\sigma_{\alpha \dot{\alpha}}{ }^{m} p_{m} \bar{\theta}^{\dot{\alpha}}\right) \\
\bar{Q}_{\dot{\alpha}} & =-i\left(\frac{\partial}{\partial \bar{\theta}^{\dot{\alpha}}}+\theta^{\alpha} \sigma_{\alpha \dot{\alpha}}{ }^{m} p_{m}\right) \\
X^{m} & =i\left(\frac{\partial}{\partial p_{m}}+\frac{p^{m} p^{n}}{\Lambda^{2}} \frac{\partial}{\partial p^{n}}\right)-\frac{1}{2} \theta \sigma^{m} \bar{\theta}+\frac{1}{2 \Lambda^{2}} \frac{\partial}{\partial \bar{\theta}} \bar{\sigma}^{m} \frac{\partial}{\partial \theta} \\
j^{m n} & =i\left(-p_{m} \frac{\partial}{\partial p_{n}}+p_{n} \frac{\partial}{\partial p_{m}}+\theta \sigma^{m n} \frac{\partial}{\partial \theta}+\frac{\partial}{\partial \bar{\theta}} \bar{\sigma}^{m n} \bar{\theta}\right)
\end{aligned}
$$

As is usual, one can construct fermionic operators $D_{\alpha}$ and $\bar{D}_{\dot{\alpha}}$ which anticommute with the super-translation generators $Q_{\alpha}$ and $\bar{Q}_{\dot{\alpha}}$, and use them to reduce supersymmetric representations. They are

$$
\begin{aligned}
& D_{\alpha}=i\left(\frac{\partial}{\partial \theta^{\alpha}}-\sigma_{\alpha \dot{\alpha}}{ }^{m} p_{m} \bar{\theta}^{\dot{\alpha}}\right) \\
& \bar{D}_{\dot{\alpha}}=-i\left(\frac{\partial}{\partial \bar{\theta}^{\dot{\alpha}}}-\theta^{\alpha} \sigma_{\alpha \dot{\alpha}}{ }^{m} p_{m}\right),
\end{aligned}
$$

satisfying

$$
\left\{D_{\alpha}, \bar{D}_{\dot{\alpha}}\right\}=-2 \sigma_{\alpha \dot{\alpha}}{ }^{m} p_{m} \quad\left\{D_{\alpha}, D_{\beta}\right\}=\left\{\bar{D}_{\dot{\alpha}}, \bar{D}_{\dot{\beta}}\right\}=0
$$

A chiral field $\Phi$ on super-momentum space satisfies

$$
\bar{D}_{\dot{\alpha}} \Phi=0
$$

This is solved by

$$
\Phi=\mathcal{F}(p, \theta) e^{-\theta p \cdot \sigma \bar{\theta}}
$$

Assuming $\mathcal{F}(p, \theta)$ to be a bosonic field, it can be expanded in terms of two bosonic component fields and a spinor femionic field. We define the action of an operator $\mathcal{O}$ on the function $\mathcal{F}(p, \theta)$ according to $\mathcal{O} \Phi=[\mathcal{O F}] e^{-\theta p \cdot \sigma \bar{\theta}}$. Applying the super-translation generators, one gets

$$
\begin{aligned}
Q_{\alpha} \mathcal{F} & =i \frac{\partial \mathcal{F}}{\partial \theta^{\alpha}} \\
\bar{Q}_{\dot{\alpha}} \mathcal{F} & =-2 i(\theta \sigma \cdot p)_{\dot{\alpha}} \mathcal{F}
\end{aligned}
$$


Similar considerations can be made for anti-chiral fields $\bar{\Phi}$, which satisfy

$$
D_{\alpha} \bar{\Phi}=0
$$

It is solved by

$$
\bar{\Phi}=\overline{\mathcal{F}}(p, \bar{\theta}) e^{\theta p \cdot \sigma \bar{\theta}}
$$

If we define the action of an operator $\mathcal{O}$ on the function $\overline{\mathcal{F}}(p, \bar{\theta})$ by $\mathcal{O} \bar{\Phi}=[\mathcal{O} \overline{\mathcal{F}}] e^{\theta p \cdot \sigma \bar{\theta}}$, then in contrast (6.7),

$$
\begin{aligned}
Q_{\alpha} \overline{\mathcal{F}} & =2 i(p \cdot \sigma \bar{\theta})_{\alpha} \overline{\mathcal{F}} \\
\bar{Q}_{\dot{\alpha}} \overline{\mathcal{F}} & =-i \frac{\partial \overline{\mathcal{F}}}{\partial \bar{\theta}^{\dot{\alpha}}}
\end{aligned}
$$

\section{Concluding remarks}

Our derivation of the supersymmetric Snyder algebra closely follows Snyder's work.[1] In particular, it uses his projection to Minkowski space-time. Other projections from de Sitter space have been considered.[5] Snyder's algebra also has been derived starting from relativistic particle dynamics.[8], [9],[10],[11],[12],[13] Particle dynamics on Snyder space has also been studied in [14],[15]. Ref. [13], in particular, begins from the reparametrization invariant action of a relativistic particle. One arrives at the Snyder algebra (or more precisely, its three-dimensional Euclidean subalgebra) from a particular gauge condition which fixed the reparametrization freedom. It should also be possible to obtain the supersymmetric Snyder algebra starting from an action principle for supersymmetric particles, for example [16]. As the system has two first class constraints it will require to gauge constraints to eliminate all gauge degrees of freedom. There may exist a choice of conditions whereby the supersymmetric Snyder algebra is realized by the Dirac brackets.

Finally, a number of generalizations of our construction are possible. One can consider supersymmetric Snyder algebras in different space-time dimensions and also extended supersymmetry. For the three-dimensional Euclidean version of the supersymmetry Snyder algebra, see [17]. Concerning extended supersymmetry, its construction is straightforward. For this we can introduce $2 N$ de Sitter spinors $\Theta_{\mathrm{A}}^{\mathrm{a}}$ and $\bar{\Theta}_{\mathrm{A}}^{\mathrm{a}}, \mathrm{a}=1, \ldots N$, satisfying

$$
\begin{aligned}
\left\{\Theta_{\mathrm{A}}^{\mathrm{a}}, \bar{\Theta}_{\mathrm{B}}^{\mathrm{b}}\right\} & =-i \delta_{\mathrm{AB}} \delta^{\mathrm{ab}} \\
\left\{\bar{\Theta}_{\mathrm{A}}^{\mathrm{a}}, \bar{\Theta}_{\mathrm{B}}^{\mathrm{b}}\right\} & =\frac{2}{\Lambda} E_{\mathrm{AB}}^{+} Z_{+}^{\mathrm{ab}} \\
\left\{\Theta_{\mathrm{A}}^{\mathrm{a}}, \Theta_{\mathrm{B}}^{\mathrm{b}}\right\} & =\frac{2}{\Lambda} E_{\mathrm{AB}}^{-} Z_{-}^{\mathrm{ab}},
\end{aligned}
$$

where $Z_{+}^{\mathrm{ab}}=-Z_{+}^{\mathrm{ba}}$ and $Z_{-}^{\mathrm{ab}}=-Z_{-}^{\mathrm{ba}}$ are central charges and we define

$$
E^{+}=\left(\begin{array}{cc}
0 & 0 \\
0 & -\epsilon_{\dot{\alpha} \dot{\beta}}
\end{array}\right) \quad E^{-}=\left(\begin{array}{cc}
\epsilon_{\alpha \beta} & 0 \\
0 & 0
\end{array}\right)
$$


The de Sitter generators (3.2) now contain a sum over $\mathrm{a}=1, \ldots N$. Upon writing

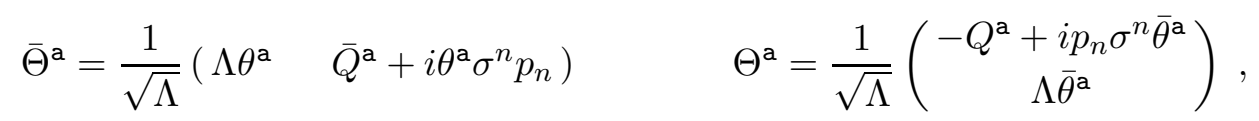

and substituting into (7.1), we recover the extended supersymmetry algebra

$$
\begin{aligned}
\left\{Q_{\alpha}^{\mathrm{a}}, \bar{Q}_{\dot{\alpha}}^{\mathrm{b}}\right\} & =2 \sigma_{\alpha \dot{\alpha}}{ }^{m} p_{m} \delta^{\mathrm{ab}} \\
\left\{Q_{\alpha}^{\mathrm{a}}, Q_{\beta}^{\mathrm{b}}\right\} & =2 \epsilon_{\alpha \beta} Z_{-}^{\mathrm{ab}} \\
\left\{\bar{Q}_{\dot{\alpha}}^{\mathrm{a}}, \bar{Q}_{\dot{\beta}}^{\mathrm{b}}\right\} & =-2 \epsilon_{\dot{\alpha} \dot{\beta}} Z_{+}^{\mathrm{ab}},
\end{aligned}
$$

upon projecting to Minkowski space-time. From (7.1), it also follows that $\theta^{\mathrm{a} \alpha}$ and $\bar{\theta}^{\mathrm{a} \dot{\alpha}}$ are Grassmann odd variables, which are canonically conjugate, respectively, to $Q_{\alpha}^{\mathrm{a}}$ and $\bar{Q}_{\dot{\alpha}}^{\mathrm{a}}$, i.e.,

$$
\left\{Q_{\alpha}^{\mathrm{a}}, \theta^{\beta^{\mathrm{b}}}\right\}=i \delta_{\alpha}^{\beta} \delta^{\mathrm{ab}} \quad\left\{\bar{Q}_{\dot{\alpha}}^{\mathrm{a}}, \bar{\theta}^{\dot{\beta} \mathrm{b}}\right\}=-i \delta_{\dot{\alpha}}^{\dot{\beta}} \delta^{\mathrm{ab}} \quad\left\{Q_{\alpha}^{\mathrm{a}}, \bar{\theta}^{\dot{\beta} \mathrm{b}}\right\}=\left\{\bar{Q}_{\dot{\alpha}}^{\mathrm{a}}, \theta^{\beta \mathrm{b}}\right\}=0
$$

\section{Acknowledgments}

L. G. was supported by the High Energy Section of ICTP. A.S. was supported in part by the DOE, Grant No. DE-FG02-10ER41714.

\section{REFERENCES}

[1] H. S. Snyder, "Quantized space-time," Phys. Rev. 71, 38 (1947).

[2] L. Lu and A. Stern, "Snyder space revisited," Nucl. Phys.B 854, 894 (2012).

[3] For a recent review of supersymmetric Yang-Mills theory on the lattice see A. Joseph, "Supersymmetric Yang-Mills theories with exact supersymmetry on the lattice," arXiv:1110.5983 [hep-lat].

[4] A. Matusis, L. Susskind and N. Toumbas, "The IR/UV connection in the non-commutative gauge theories," JHEP 0012, 002 (2000); H. O. Girotti, M. Gomes, V. O. Rivelles and A. J. da Silva, "A consistent noncommutative field theory: The Wess-Zumino model," Nucl. Phys. B 587, 299 (2000); "The noncommutative supersymmetric nonlinear sigma model," Int. J. Mod. Phys. A 17, 1503 (2002); F. R. Ruiz, "Gauge-fixing independence of IR divergences in non-commutative $\mathrm{U}(1)$, perturbative tachyonic instabilities and supersymmetry," Phys. Lett. B 502, 274 (2001).

[5] M. Hatsuda, W. Siegel, "Snyderspace," Nucl. Phys. B681, 152-162 (2004).

[6] S. J. Gates, M. T. Grisaru, M. Rocek, W. Siegel, "Superspace Or One Thousand and One Lessons in Supersymmetry," Front. Phys. 58, 1-548 (1983). 
[7] J. Wess and J. Bagger, "Supersymmetry and supergravity," Princeton, USA: Univ. Pr. (1992) $259 p$.

[8] G. Jaroszkiewicz, "A dynamical model for the origin of Snyder's quantized spacetime algebra," J. Phys. A: Math. Gen. 28 L343 (1995).

[9] J. M. Romero and A. Zamora, "Snyder noncommutative space-time from two-time physics," Phys. Rev. D 70, 105006 (2004).

[10] J. M. Romero and J. D. Vergara, "The Parametrized relativistic particle and the Snyder space-time," arXiv:hep-th/0602058.

[11] R. Banerjee, S. Kulkarni and S. Samanta, "Deformed symmetry in Snyder space and relativistic particle dynamics," JHEP 0605, 077 (2006).

[12] C. Chatterjee and S. Gangopadhyay, "kappa-Minkowski and Snyder algebra from reparametrisation symmetry," Europhys. Lett. 83, 21002 (2008).

[13] A. Stern, "Relativistic Particles on Quantum Space-time," Phys. Lett. A 375, 2498 (2011).

[14] S. Mignemi, "Classical and quantum mechanics of the nonrelativistic Snyder model," Phys. Rev. D84, 025021 (2011); "Classical and quantum mechanics of the nonrelativistic Snyder model in curved space," [arXiv:1110.0201 [hep-th]].

[15] L. Lu and A. Stern, "Particle Dynamics on Snyder space," arXiv:1110.4112 [hep-th].

[16] C. A. P. Galvao, C. Teitelboim, J. Math. Phys. 21, 1863 (1980).

[17] A. Stern, "Properties of Snyder space," to appear in Int. J. of Geometric Methods in Modern Physics. 